\title{
BMJ Open Photoaging smartphone app promoting poster campaign to reduce smoking prevalence in secondary schools: the Smokerface Randomized Trial: design and baseline characteristics
}

Titus J Brinker, ${ }^{1}$ Julia Holzapfel, ${ }^{1}$ Tanja G Baudson, ${ }^{2}$ Katharina Sies, ${ }^{3}$ Lena Jakob, ${ }^{3}$ Hannah Maria Baumert, ${ }^{3}$ Marlene Heckl, ${ }^{4}$ Ana Cirac, ${ }^{4}$ Janina L Suhre, ${ }^{5}$ Verena Mathes, ${ }^{1}$ Fabian N Fries, ${ }^{6}$ Hannah Spielmann, ${ }^{6}$ Nancy Rigotti, ${ }^{7}$ Werner Seeger, ${ }^{1}$ Felix Herth, ${ }^{8}$ David A Groneberg, ${ }^{9}$ Tobias Raupach, ${ }^{10}$ Henning Gall, ${ }^{1}$ Claudia Bauer, ${ }^{8}$ Pat Marek, ${ }^{11}$ Anil Batra, ${ }^{12}$ Chase H Harrison, ${ }^{13}$ Lava Taha, ${ }^{14}$ Andreas Owczarek, ${ }^{1}$ Felix J Hofmann, ${ }^{1}$ Roger Thomas, ${ }^{15}$ Ute Mons, ${ }^{16}$ Michael Kreuter ${ }^{8}$

To cite: Brinker TJ, Holzapfel J, Baudson TG, et al. Photoaging smartphone app promoting poster campaign to reduce smoking prevalence in secondary schools: the Smokerface Randomized Trial: design and baseline characteristics. BMJ Open 2016;6:e014288. doi:10.1136/bmjopen-2016014288

- Prepublication history and additional material is available. To view please visit the journal (http://dx.doi.org/ 10.1136/bmjopen-2016014288).

Received 14 September 2016 Accepted 29 September 2016

CrossMark

For numbered affiliations see end of article.

Correspondence to

Titus J Brinker;

titus.brinker@gmail.com

\section{ABSTRACT}

Introduction: Smoking is the largest cause of preventable death globally. Most smokers smoke their first cigarette in early adolescence. We took advantage of the widespread availability of mobile phones and adolescents' interest in appearance to develop a free photoaging app which is promoted via a poster campaign in secondary schools. This study aims to evaluate its effectiveness regarding smoking prevalence and students' attitudes towards smoking.

Methods and analysis: A randomised controlled trial is conducted with 9851 students of both genders with an average age of 12 years in grades 6 and 7 of 126 secondary schools in Germany. At present, cigarette smoking prevalence in our sample is $4.7 \%$, with $4.6 \%$ of the students currently using e-cigarettes (1.6\% use both). The prospective experimental study design includes measurements at baseline and at 6,12 and 24 months postintervention via a questionnaire plus a random cotinine saliva sample at 24 months postintervention. The study groups consist of randomised schools receiving the Smokerface poster campaign and control schools with comparable baseline data (no intervention). The primary end point is the difference of change in smoking prevalence in the intervention group versus the difference in the control group at 24 months follow-up. Longitudinal changes in smoking-related attitudes, the number of new smokers and quitters and the change in the number of neversmokers will be compared between the two groups as secondary outcomes.

Ethics and dissemination: Ethical approval was obtained from the ethics committee of the University of Gießen and the ministries of cultural affairs, both in Germany. Results will be disseminated at conferences, in peer-reviewed journals, on our websites and throughout the multinational Education Against Tobacco network.

\section{Strengths and limitations of this study}

- This is the first randomised trial on app-based tobacco prevention in the school setting.

- The majority of German 12-year-old pupils use smartphones on a regular basis.

- The campaign is easy to implement, may be added to existing campaigns and costs $<€ 50$ per school.

- A random cotinine saliva sample is obtained for biochemical validation.

- The large sample and the method of data collection provide valuable epidemiological data on adolescent smoking in Germany.

Trial registration number: NCT02544360, Pre-results.

\section{INTRODUCTION}

Smoking is a risk factor for various diseases, leading to the highest number of avoidable deaths in Germany and worldwide. ${ }^{1}$ Most smokers start smoking in early adolescence ${ }^{2}$ and relatively few of those who become regular smokers manage to quit later in life. ${ }^{3}$ The 2016 HBSC international report by the WHO revealed that $15 \%$ of boys and $13 \%$ of girls aged 15 years claimed to have smoked at least once a week in Germany in $2013 / 2014 .^{3}$ In spite of the decline in prevalence over recent years, strong socioeconomic and educational inequalities in smoking exist. ${ }^{3-5}$ 
Many effective school-based interventions that prevent smoking are based on external mentors and thus not broadly available. ${ }^{6}$ This limitation is often accompanied by high costs or organisational effort, which reduces practical implementation in schools. ${ }^{7}$

Photoaging desktop programs in which an image is altered to predict future appearance have been shown to help motivate women aged 14-18 years to quit smoking and increased the quit rate in young adults aged 18-30 years of both genders by $21 \% .^{8} 9$ However, these computer programs only reach a small audience and are not freely available.

We took advantage of the broad availability of smartphones and adolescents' interest in appearance to create the free photoaging smartphone app 'Smokerface'. We demonstrated its implementation in a small cross-sectional pilot study via a technique called mirroring: The students' altered three-dimensional selfportraits on mobile phones or tablets were 'mirrored' via a projector in front of their whole grade. ${ }^{10}$ Our pilot study showed that the app motivates German adolescents not to smoke. However, the method of implementation used in the pilot study cost $€ 2000$ per school, requires the time of health educators and longitudinal effects were not measured. In this trial, the photoaging app is implemented in secondary schools via a low cost $€ 50$ per school) and widespread poster campaign.

We designed this randomised trial to answer the following main questions: Is this low-cost campaign effective in convincing students to use the app? Which students are more likely to use an app revealing the photoaging effects of smoking? How does this campaign or the use of the app affect smoking prevalence among the students or change their attitudes towards smoking in accordance with the Theory of Planned Behavior? ${ }^{11}$

\section{METHODS}

\section{Trial design}

The Smokerface trial is designed as a randomised, controlled superiority multicentred trial with two parallel groups and a primary end point of the difference in smoking prevalence from baseline to follow-up compared between the two groups (figure 1). Randomisation was externally and centrally performed at school level with a 1:1 allocation.

A total of 126 secondary schools in 6 federal states (details: authors' contributions) of Germany participated in the baseline survey in the first half of the school year (October 2015-April 2016 depending on the federal state) prior to randomisation. The randomisation of schools based on the baseline data was completed from February through 1 May 2016. Immediately after randomisation, the posters were hung by trained data collectors from March through June of 2016. The first follow-up survey will be 6 months thereafter. At 1-year follow-up (March-June 2017) and at 2-year follow-up (March-June 2018), the third and fourth
Recruitment of pupils attending grades six and seven of regular secondary schools.

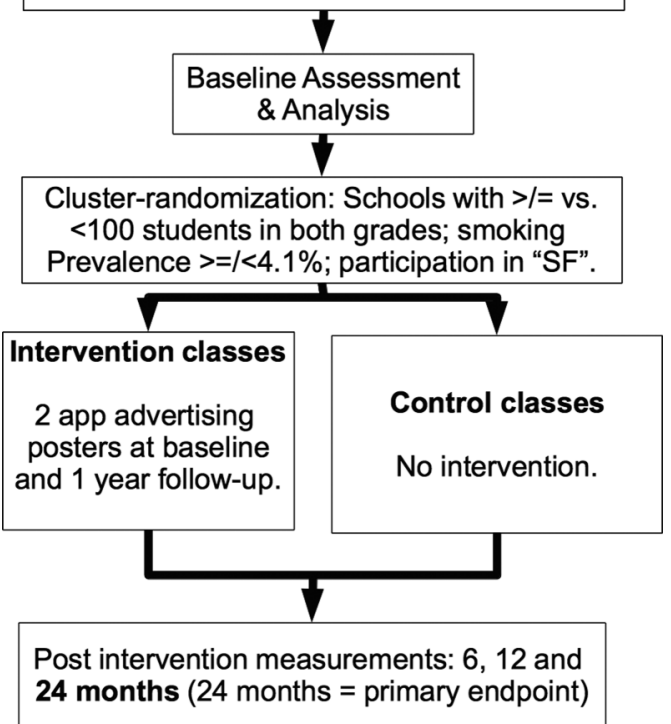

Figure 1 Study design.

waves of the survey will be conducted. At 1 year follow-up, additional posters are hung (see Intervention section for details).

\section{Intervention}

The posters

Two standardised DIN A1 (a size of $594 \times 841 \mathrm{~mm}$ ) posters advertising the app (figures $3 \mathrm{~A}, \mathrm{~B}$ ) are both hung up in at least $50 \%$ of rooms where pupils of grades 6 and 7 attend classes. The posters include large images of the short-term photoaging effects of smoking ( 1 pack a day for 1 year) and small images of the long-term effects (1 pack a day for 15 years). The posters illustrate brittle hair and earlier hair loss due to smoking, ${ }^{12} 13$ a higher risk of acne and associated pimples, ${ }^{14}{ }^{15}$ wrinkles, larger pores, pale skin, yellow teeth ${ }^{13} 16$ and a higher risk of a runny nose (common colds), which also affects outward appearance negatively. The models are both male and female and appear to be about 17 years old, so that they are perceived as role models by both genders of our young target group. The posters are in German (English translation for publication purposes). In the corner of each poster, pupils are asked to find out what their faces would look like as smokers by downloading the free Smokerface app.

A small pilot study with 91 pupils identified that $89 / 91$ $(97.8 \%)$ noticed the posters but only about $20 \%$ of them realised that there was a free app (first version; figure 2A, B). Therefore, we optimised the posters to provide a stronger call to action and added a $\mathrm{QR}$ code (figure 3A, B).

\section{Letters to students, teachers and parents}

To further increase the use of the app within the intervention group, the intervention posters will be 

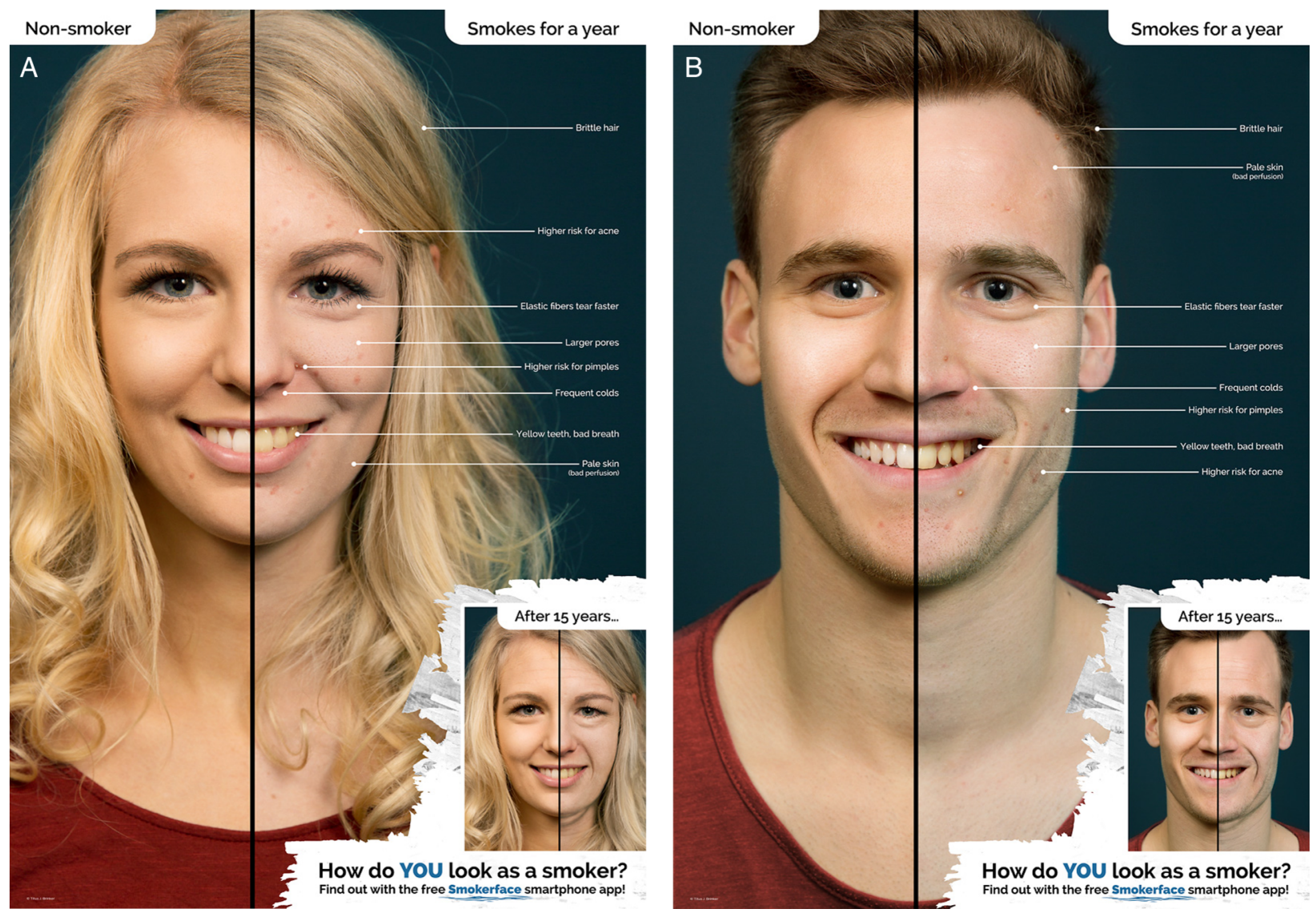

complemented by letters to the students, teachers and parents (delivered along with the posters and at the 6 and 12 months follow-up) which explain the photoaging effects of smoking and encourage app use. Teachers and parents are also encouraged to use the app with their students and all smokers are provided quit advice (brief quit-advice and free motivational cessation app 'Smokerstop').

\section{Raffle and change of posters at 12 months follow-up}

To further increase adherence to the app in a way that does not confound the effects of the original intervention, a raffle is implemented directly after the 1 year follow-up. Students are informed via letter that they have the chance to win the newest version of the iPhone (Apple, California, USA) if they email us their Smokerface image via the app.

Second, additional posters featuring long-term smoking consequences are hung in the classrooms at 12-month follow-up, where the small preview face and the large face have been swapped (figure $4 \mathrm{~A}, \mathrm{~B}$ ). This is performed in the same manner as with the posters at baseline. The purpose of this is to further increase the number of app users and to counteract the diminishing salience of the original posters to the students over time, a process which has been described in detail with graphic health warnings. ${ }^{17}$ However, since no selfie looks the same, we expect that at least the pupils who installed the app do not get used to the effects as fast as this is the case with graphic health warnings.

\section{The associated photoaging app 'Smokerface'}

The app photoages the user's own face into an animated future smoker's face (after 1, 3, 6, 9, 12, 15 years of smoking one pack a day) and contrasts the image with the animated future face as a non-smoker ${ }^{18}$ (see online supplementary video 1). Furthermore, the app explains all the positive effects of not smoking on an image of a non-smoker who appears youthful compared to the negative effects of smoking on a prematurely aged face. ${ }^{18}$ The app is freely available in the six most spoken languages worldwide.

\section{Monitoring}

Posters

Our trained data collectors are required to personally hang the posters and record this procedure in detail. Additional posters are provided to the person responsible for each school (mostly teachers) so that they can replace posters in their schools that are vandalised or ripped down. During the first 12 months, each intervention school will receive a bimonthly email reminder asking how many of the posters are still hanging or have been replaced. At the 6,12 and 24 months follow-ups, the percentage of posters still available and intact is checked and noted by our trained data collectors. In 

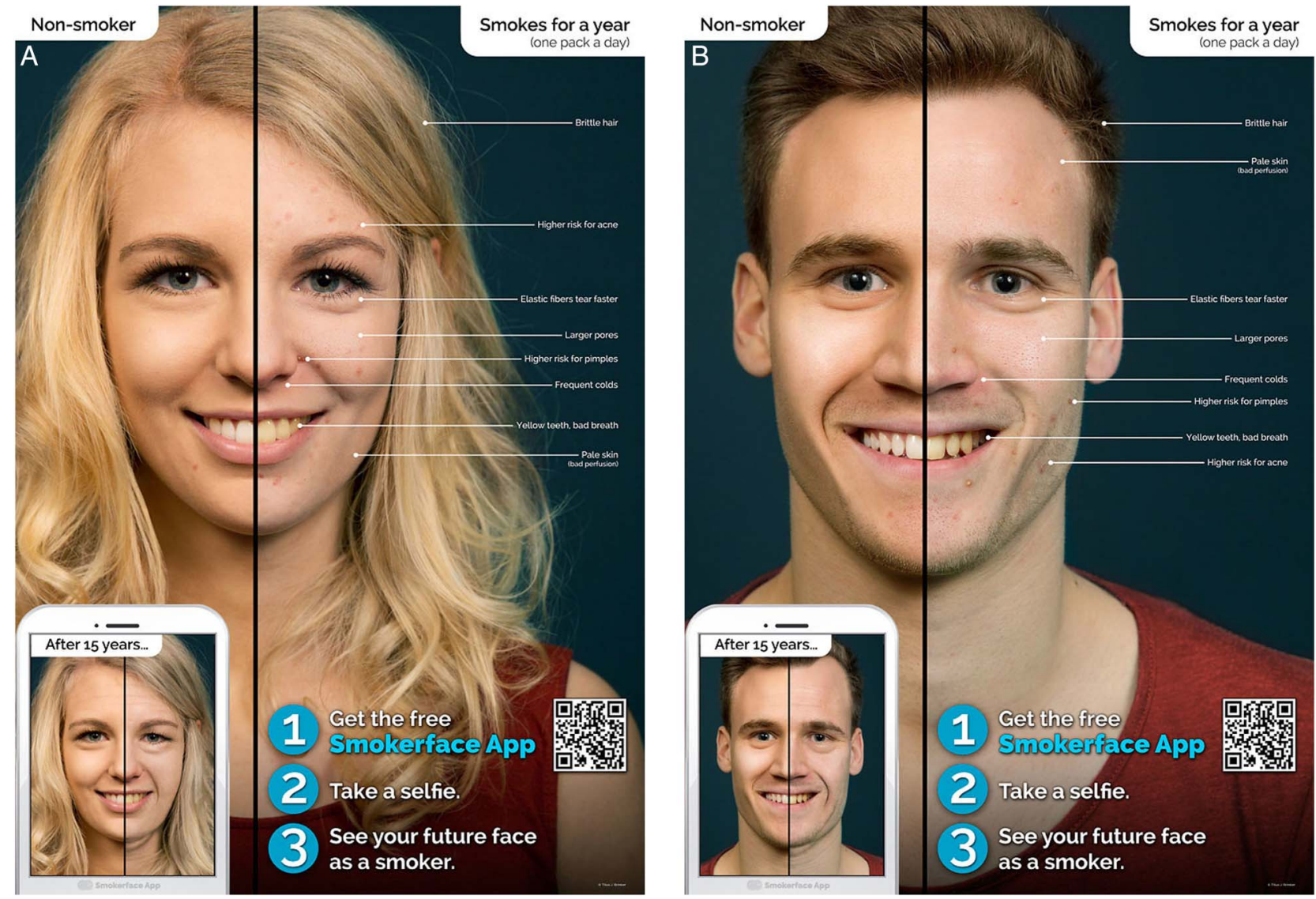

Figure 3 (A): Female version of the optimised Smokerface poster. (B): Male version of the optimised Smokerface poster.

addition, our questionnaire for the intervention group includes an item asking the pupils the quantity of posters still hanging in their school (all, more than half, less than half, none, do not know). To avoid bias through changed classrooms in a new school year, additional prospective classrooms will be targeted by the campaign.

\section{App stability}

The stability of the Smokerface app is monitored during the full study period via the application Crashlytics (San Francisco, California, USA) to ensure that the app stably operates on the majority of smartphones.

\section{Participants}

\section{Eligibility criteria at baseline}

Students from Germany attending grades 6 and 7 in all types of regular secondary school in Germany were eligible. Schools of other types or countries, or schools that had previously participated in an Education Against Tobacco event where the Smokerface app was presented, were not eligible (educationtobacco.org).

\section{Contaminated classes}

All classes will be included in the final intention-to-treat analysis even if some control-arm classes are contaminated with some students who used the app. However, app use will be assessed in both groups at the end point and will be the basis for a secondary (sensitivity) analysis with the methods described in the Analysis section of this protocol.

\section{Procedure}

Data are collected via a questionnaire and a random cotinine saliva sample. In addition to sociodemographic data (age, gender and school type), the questionnaire captures the smoking status of the school students concerning cigarettes and multiple other tobacco products (such as e-cigarettes and water pipes). These items are based on three established studies ${ }^{19-21}$ and were either used in their original form or adapted to the specific circumstances of the present study. Since no German equivalents of the instruments were available, we used the Conceptual Method for translation described by the WHO/UNESCAP Project on Health and Disability Statistics. ${ }^{22}$ Newly translated and/or modified materials were extensively pretested and subjected to statistical analyses (internal consistency/Cronbach's $\alpha$, exploratory and confirmatory factor analyses, which represented the basis for item selection. Details about this procedure are documented in the online supplementary material.

\section{Biochemical validation}

Cotinine saliva samples will be obtained from all students who provide active written consent themselves and parental consent at 18 months follow-up. Analysis via 

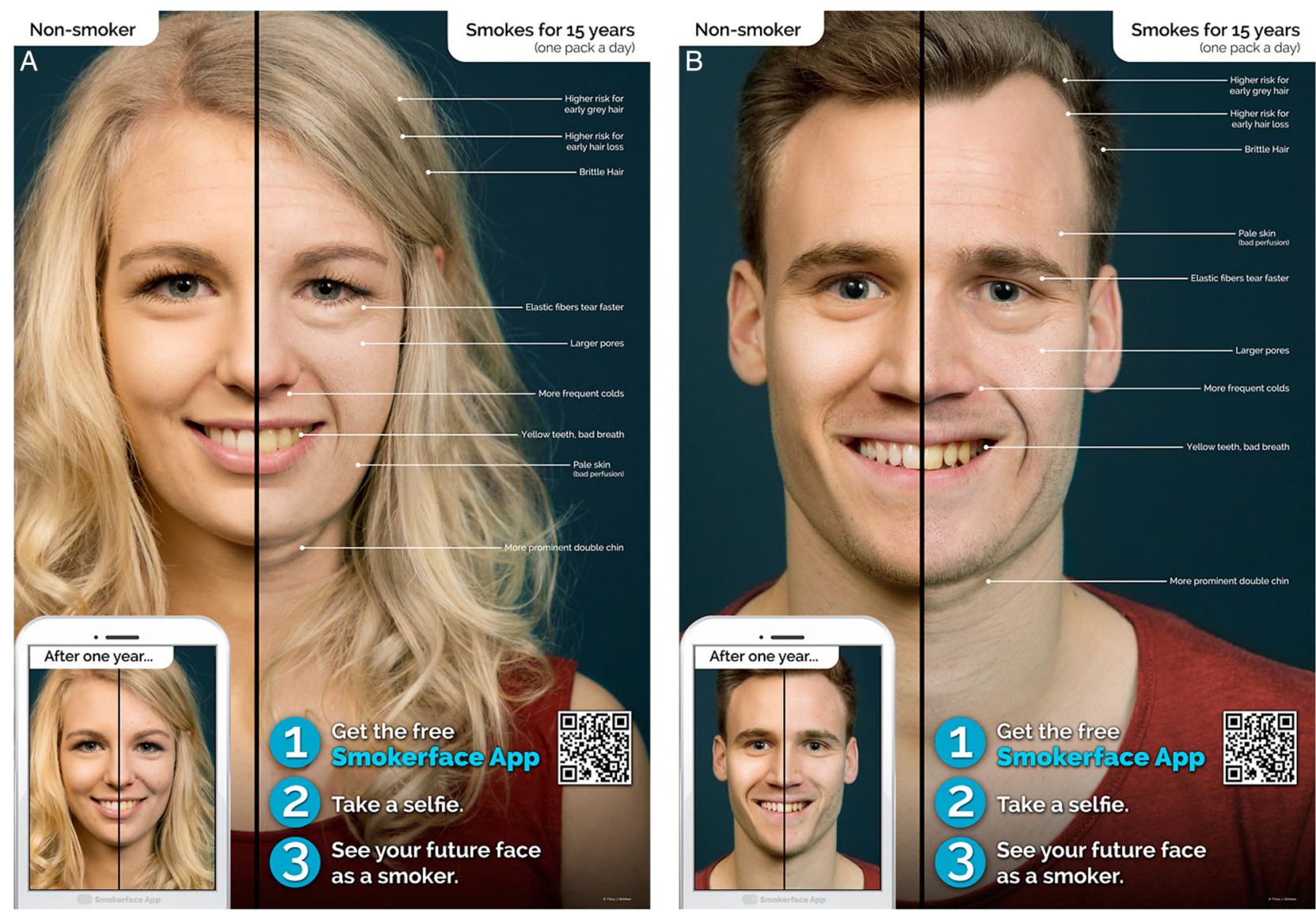

Figure 4 (A): Female version of the optimised Smokerface poster (15-year version). (B): Male version of the optimised Smokerface poster (15-year version).

liquid chromatography-mass spectrometry and therewith a validation of smoking status will be performed in a random subsample of these students. We aim at a subsample size of $10 \%$ of students with active and passive parental consent, but of at least 300 students. All samples will be destroyed immediately after their cotinine level was measured and have no other purpose.

\section{Data collection}

Each data collector received training for data collection and was required to use an adapted standardised protocol for data collection, an optimised version of the one used in the Hutchinson Smoking Prevention Project, which was discussed with and provided by the original authors. $^{19}$

\section{Cluster-randomisation}

Schools (grades 6 and 7) were externally and centrally randomly assigned to the intervention or control group in a ratio of 1:1 (deviating slightly due to the method of stratification) via computer by the Center for Clinical Trials (KKS) in Marburg, Germany. The smoking prevalence within grade 7 of the school $(>4.1 \% / \leq 4.1 \%)$, the school size (>100 pupils/ $\leq 100$ pupils) and the participation in 'Smokefree' (SF; participation vs no participation) were considered as factors for stratification.

\section{Smokefree}

In the Rhine-Neckar region, we have to consider the primary smoking intervention group 'Smokefree' (SF; German: 'ohnekippe'), which takes place in 32 schools included from this area, ${ }^{23}$ as a confounder. $\mathrm{SF}$ is a 2 hour informational event for 7 th graders taking place in a specialised chest clinic which uses a classic fear approach aiming to reduce smoking prevalence. Since not all schools within our sample participate in the SF event, stratification is required.

\section{Outcomes}

The primary end point is defined as the difference of the change in smoking prevalence from baseline to 24-month follow-up between the two groups. The longitudinal change in smoking-related attitudes in accordance with the Theory of Planned Behavior, the number of new smokers, the number of quitters and the change in the number of never-smokers after 24 months will be compared between the two groups as secondary outcomes. For all end points, the number needed to treat (NNT) will be calculated in addition. Considering the low costs of the intervention, we predefined a NNT below 100 as clinically relevant. A smoker is defined as a pupil who claims to have used cigarettes at least 1 day in the 30 days preceding the survey, in accordance with the established 
National Youth Tobacco Survey definition. ${ }^{15}$ Those pupils who claim not to have smoked cigarettes in the past 30 days are defined as non-smokers. All participants who claim to have smoked more than a puff in the past (beyond the past 30 days) are defined as ex-smokers.

\section{Statistical considerations}

\section{Sample size calculation}

The sample size for comparing the primary end point between the two groups was calculated with a two-sided $\chi^{2}$ test and multiplied by the correction factor $\mathrm{DE}$ (=design effect; $\mathrm{DE}=1+(\mathrm{m}-1) \times \mathrm{ICC}$; with $\mathrm{m}=$ cluster size, ICC $=$ Intraclass correlation coefficient) to adjust for correlation with regard to smoking prevalence within a cluster. We calculated an ICC of 0.033 , based on the data from our recently published study on smoking behaviour in Germany (analysis-of-variance estimator by Zou and Donner). ${ }^{24} 25$

To detect a difference of $3 \%$ in change of smoking prevalence from baseline to 24 months of follow-up between the two groups with an $\alpha$ of $5 \%$ and a test power of $70 \%$, we calculated a sample size of 564515715 participants, depending on the change of smoking prevalence of the two groups (2\% vs $5 \%$ up to $9 \%$ vs $12 \%$ ) and with an assumed dropout rate of $30 \%$ to follow-up. Assuming an average cluster size of 100 participants, 56-157 schools needed to be randomised. The dropout rate of $30 \%$ is appropriate for our 24 months follow-up, as we observed $<20 \%$ dropout in our recent 6-month investigations. ${ }^{24} 26$

\section{Data entry}

Data entry is performed with the help of the current software version of Formic Fusion by the Xerox AG (Kloten, Switzerland) and recommended scanners.

\section{Analysis}

To examine baseline differences in pupils' characteristics in our experimental design, we will use $\chi^{2}$ tests for the categorical variables and t-tests for continuous variables. To test for differences in baseline and follow-up smoking prevalence between groups, we will use a cluster-adjusted Mantel-Haenszel $\chi^{2}$ test $^{27}$ at a two-sided significance level of 5\% with 'participation in the SF event' as a stratification factor. In the main analysis, HLM (hierarchical linear models) will be applied. HLM can handle the nested structure of the data and will be used to test for between-group differences in within-group changes in smoking behaviour over time. HLM will also be used to investigate the influence of further covariates (such as gender, culture and social characteristics) and timedependent behaviour in secondary analyses. Statistical analyses will be performed using the newest version of SPSS Statistics (IBM Corporation, New York, USA).

The effect that missing data might have on results will be assessed via sensitivity analysis. Dropouts (essentially participants who withdraw consent for continued follow-up or who are missing in the classroom during the survey) will be included in the analysis by modern imputation methods for missing data. Multiple imputation will be used to estimate treatment effect. ${ }^{28}$

\section{Baseline characteristics DISCUSSION}

While the use of mobile phone technology in smoking cessation was investigated in several trials, ${ }^{29}$ this is the first major randomised trial on app-based prevention in the school setting. Photoaging mobile apps in particular have never been tested for their influence on adolescent smoking prevalence, ${ }^{18}$ although the physical self-concept plays the most important role for the self-esteem of adolescents ${ }^{30}$ and photoaging interventions have been found to increase the quit rates of young adult smokers. ${ }^{8}$ The investigated intervention is freely accessible in our online supplementary materials, easy to implement, low cost, independent from external health educators and can be added to existing school-based programmes.

\section{Baseline characteristics}

Our baseline survey includes the major predictors of adolescent smoking as described in the literature. ${ }^{21} 31$ The differences between the two groups are balanced.

This large study is enrolled in six German federal states. Our definitions for the smoking status of the various monitored tobacco products stem from the National Youth Tobacco Survey by the Centers for Disease Control (Atlanta, USA) ${ }^{21}$ Data are collected by external trained data collectors who use the same (translated) materials for training and the same data collection protocol as used in the Hutchinson Smoking Prevention Project to ensure international comparability and are validated at endline via a cotinine sample as recommended in the Cochrane Analysis. ${ }^{719}$ All baseline data were collected before the e-cigarette sales ban was implemented on 1 April 2016 in Germany. This is the first national study to show that current e-cigarette and cigarette smoking prevalence in grades 6 and 7 of secondary schools are the same $(4.7 \%$ use cigarettes and $4.6 \%$ use e-cigarettes). Almost a third of these $(1.6 \%$ of the total sample) currently use both products at the same time. ${ }^{21}$ The 6 months follow-up of this survey will also reveal the effectiveness of the e-cigarette sales ban to our very sensitive age group. The epidemiological data presented here is therefore also valuable considering that the most cited and most recent surveys in Germany were conducted via telephone interviews, a method showing poor consistency with biochemical validation in our age group. ${ }^{42}$

\section{Quality of data collection}

We monitored the quality of the data collection with the same two items used in the Hutchinson survey: (1) Was it explained to you that nobody else than the researchers would see your questionnaire? (2) Did you know the day the survey would be performed in your class? 
Table 1 Baseline characteristics

\begin{tabular}{|c|c|c|c|}
\hline Variable & Entire sample (\%) (n) & Intervention group (\%) (n) & Control group (\%) (n) \\
\hline Number of pupils & (100) 9851 & (53.2) 5238 & (46.8) 4613 \\
\hline Number of schools & (100) 126 & $(52.4) 66$ & (47.6) 60 \\
\hline Grammar Schools & $(100) 25$ & (16.7) 11 & (23.3) 14 \\
\hline Gender & (97.5) 9609 & & \\
\hline Female & (46.8) 4612 & (49.3) 2520 & (46.5) 2092 \\
\hline Male & (50.7) 4997 & $(50.7) 2592$ & (53.5) 2405 \\
\hline \multirow[t]{2}{*}{ Age } & (98.6) 9710 & (53) 5151 & (47) 4559 \\
\hline & & $M=12.01(S D=0.86)$ & $\mathrm{M}=11.98(\mathrm{SD}=0.84)$ \\
\hline Number of pupils per grade & (100) 9851 & & \\
\hline Missing in questionnaire (either grade 6 or 7 ) & (1.5) 156 & $(0.8) 44$ & (2.4) 112 \\
\hline 6 & $(49.7) 4892$ & (48.9) 2559 & $(50.6) 2333$ \\
\hline 7 & (48.8) 4803 & (50.3) 2635 & (47) 2168 \\
\hline Current cigarette smokers & $(4.7$ of 9756 & $(4.1$ of 5185 & (5.5 of 4571 with \\
\hline At least once in the past 30 days & valid answers) 463 & valid answers) 210 & valid answers) 253 \\
\hline Average days of use in the past 30 days & $\mathrm{M}=7.91(\mathrm{SD}=9.78)$ & $M=8.3(S D=9.92)$ & $\mathrm{M}=7.58(\mathrm{SD}=9.68)$ \\
\hline Average number of cigarettes smoked in the past 30 days & $M=42.91(S D=105.05)$ & $M=40.6(S D=98.6)$ & $M=44.83(S D=110.28)$ \\
\hline $1-2$ days/average cigarettes per day (acd) & (2.4) $237 / 1.25$ & (1.9) 99/1.33 & (3) $138 / 1.19$ \\
\hline $3-5$ days/acd & (0.7) 67/2.12 & (0.6) $33 / 2.52$ & $(0.7) 34 / 1.74$ \\
\hline $6-9$ days/acd & (0.5) $44 / 3.08$ & (0.4) 23/3.04 & (0.5) 21/3.12 \\
\hline 10-19 days/acd & $(0.4) 39 / 5.31$ & (0.4) 20/4.92 & $(0.4) 19 / 5.71$ \\
\hline 20-29 days/acd & (0.3) 25/4.34 & (0.2) 9/3.33 & (0.4) $16 / 4.91$ \\
\hline All 30 days/acd & (0.5) $51 / 7.88$ & (0.5) $26 / 6.88$ & (0.5) 25/8.88 \\
\hline Not in the past 30 days (=non-smokers) & (95.3) 9293 & (95.9) 4975 & $(94.5) 4318$ \\
\hline I never tried smoking, not even a puff & $\begin{array}{l}(80.2) 7755 \\
\text { ( } n=9672 \text { valid answers) }\end{array}$ & (80.5) 4145 & (79.9) 3610 \\
\hline Never smoked a cigarette (never-smokers) & $\begin{array}{l}\text { (94.1 of } 9748 \\
\text { valid answers) } 9175\end{array}$ & (95.2) 4931 & (92.9) 4244 \\
\hline \multicolumn{4}{|l|}{ Ex-smokers who smoked } \\
\hline More than once per week & (1.8) 175 & $(1.4) 72$ & (2.3) 103 \\
\hline Less than once per week & (1.8) 177 & (1.5) 77 & (2.2) 100 \\
\hline \multicolumn{4}{|l|}{ Average age of first puff (years) } \\
\hline 8 years or younger & (11.3) 215 & (12.6) 126 & (9.8) 89 \\
\hline $9-10$ & (20.1) 384 & (19.9) 200 & (20.3) 184 \\
\hline $11-12$ & (57.2) 1092 & (55.9) 561 & (58.6) 531 \\
\hline $13-14$ & (11.4) 218 & (11.6) 116 & (11.3) 102 \\
\hline $\begin{array}{l}\text { Intention to smoke cigarettes }(0-6) \\
\quad(0=I \text { am very sure that I will never smoke; } 6=\mid \text { believe that } \\
\text { I will start smoking within the next month) }\end{array}$ & 0.51 & 0.49 & 0.53 \\
\hline $\begin{array}{l}\text { Do you intend to quit cigarettes? }(0-3) \\
(0=\text { No; } 3=\text { within the next month) }\end{array}$ & 0.84 & 0.71 & 0.97 \\
\hline $\begin{array}{l}\text { Current tobacco waterpipe smokers/average days of } \\
\text { use in the past } 30 \text { days (ad30) }\end{array}$ & $\begin{array}{l}\text { (2.3) } 228 / \mathrm{M}=6.73 \\
(\mathrm{SD}=9.2)\end{array}$ & $\begin{array}{l}(2.4) 125 / \mathrm{M}=6.93 \\
(\mathrm{SD}=9.34)\end{array}$ & $\begin{array}{l}(2.3) 103 / \mathrm{M}=6.49 \\
(\mathrm{SD}=9.08)\end{array}$ \\
\hline
\end{tabular}


Table 1 Continued

\begin{tabular}{|c|c|c|c|}
\hline Variable & Entire sample (\%) (n) & Intervention group (\%) (n) & Control group (\%) (n) \\
\hline Current e-cigarette smokers/ad30 & (4.6) $443 / \mathrm{M}=6.25(\mathrm{SD}=8.61)$ & (4.6) $237 / \mathrm{M}=6.24(\mathrm{SD}=8.51)$ & (4.5) $206 / \mathrm{M}=6.27(\mathrm{SD}=8.75)$ \\
\hline Current cigar or cigarillo smokers/ad30 & (0.6) $56 / \mathrm{M}=6.87(\mathrm{SD}=9.81)$ & (0.6) $33 / \mathrm{M}=7.76(\mathrm{SD}=9.89)$ & (0.5) $23 / \mathrm{M}=5.59(\mathrm{SD}=9.76)$ \\
\hline Current chewing tobacco consumers/ad30 & (0.2) $17 / \mathrm{M}=8.56(\mathrm{SD}=11.1)$ & $(0.2) 8 / \mathrm{M}=11(\mathrm{SD}=12.58)$ & $(0.2) 9 / M=6.39(S D=9.83)$ \\
\hline Current Marihuana smokers/ad30 & $(0.8) 78 / \mathrm{M}=7.67(\mathrm{SD}=10.75)$ & (0.9) $45 / \mathrm{M}=8.22(\mathrm{SD}=11.27)$ & (0.7) $33 / \mathrm{M}=6.91(\mathrm{SD}=10.12)$ \\
\hline Current steam stone waterpipe smokers/ad30 & (1.7) $168 / \mathrm{M}=6.13(\mathrm{SD}=8.59)$ & (1.9) $102 / \mathrm{M}=5.94(\mathrm{SD}=8.39)$ & (1.4) $66 / \mathrm{M}=6.41(\mathrm{SD}=8.95)$ \\
\hline Current use of other tobacco product/ad30 & $(0.7) 71 / \mathrm{M}=8.18(\mathrm{SD}=10.39)$ & (0.7) $35 / \mathrm{M}=10.31(\mathrm{SD}=11.89)$ & (0.8) $36 / \mathrm{M}=6.1(\mathrm{SD}=8.34)$ \\
\hline Current use of two tobacco products & (3.6) 351 & (3.6) 187 & (3.6) 164 \\
\hline Current use of e-cigarettes and cigarettes & (1.6) 160 & $(1.5) 78$ & (1.8) 82 \\
\hline Current use of waterpipe with tobacco and cigarettes & (1.0) 101 & (1.0) 51 & (1.1) 50 \\
\hline \multicolumn{4}{|l|}{ Ex-smokers who smoked } \\
\hline More than once per week & (1.8) 175 & $(1.4) 72$ & (2.3) 103 \\
\hline Less than once per week & (1.8) 177 & (1.5) 77 & (2.2) 100 \\
\hline I have at least one smoking parent & (48.6) 4595 & $(47.7) 2386$ & $(49.7) 2209$ \\
\hline One of my best friends smokes & (15.8) 1413 & (15) 717 & (16.6) 696 \\
\hline I have an older sibling that smokes & (15.3) 1422 & (13.7) 675 & (17.1) 747 \\
\hline \multicolumn{4}{|l|}{ Migrational background } \\
\hline Both parents born in Germany & (69.6) 6721 & (66.3) 3403 & (73.3) 3318 \\
\hline One parent born in Germany & (13.1) 1263 & (13.4) 689 & (12.7) 574 \\
\hline No parent born in Germany & (17.3) 1670 & (20.2) 1038 & (14) 632 \\
\hline \multirow[t]{2}{*}{ School performance (self-reported point average) } & (96) 9460 & (53) 5011 & (47) 4449 \\
\hline & & $\mathrm{M}=2.44(\mathrm{SD}=0.79)$ & $\mathrm{M}=2.45(\mathrm{SD}=0.8)$ \\
\hline \multicolumn{4}{|c|}{ Educational level (1-5) ( $1=$ no completed school education; $5=$ completed university) } \\
\hline Father & 3.62 & 3.58 & 3.67 \\
\hline Mother & 3.6 & 3.57 & 3.64 \\
\hline \multicolumn{4}{|l|}{ 'Do you live in the same household with your parents?' } \\
\hline I live with no parent & (5) 488 & (5) 262 & (4.9) 226 \\
\hline I live with one parent & $(24.5) 2408$ & (23.8) 1247 & $(25.1) 1161$ \\
\hline I live with both parents & (70.6) 6955 & (71.2) 3729 & (69.9) 3226 \\
\hline Student participation rate & (69.54) $9851 / 14165$ & (67.33) 5238/7780 & (72.25) $4613 / 6385$ \\
\hline 'I know that this survey is anonymous and what that means'. & (88.4) 8520 & $(87.7) 4475$ & (89.2) 4045 \\
\hline 'I knew that the survey would be today'. & (32.6) 3121 & (34.9) 1770 & (30) 1351 \\
\hline
\end{tabular}


Item (1) was asked at the end of the questionnaire to monitor whether the data collectors had explained this fact in detail (and the students remember it) and (2) was asked to monitor if the students had been able to mentally prepare for the survey, which is considered as a bias in school-based research which we tried to avoid based on communications with the responsible teachers. ${ }^{19}$ About $88.4 \%$ of the students remembered at the end of the questionnaire that the data collectors had explained the confidentiality and only $32.6 \%$ of students knew the day the survey would be conducted.

Since we were obliged to obtain active consent from the parents and the students, almost all students who originally agreed to participate in the survey and had obtained parental consent eventually participated (69.54\% of all students in the schools under investigation; see table 1). A small fraction of students was not present in the classroom when the trained data collectors visited them (on average about 2 participants per class), ${ }^{18}{ }^{26}$ which cannot be avoided when external data collectors are used.

\section{Generalisability}

Since this study was conducted only in Germany, results might not be generalisable to other cultural or national settings. However, cosmetic products are used by adolescents in the majority of countries and the WHO is reportedly concerned about the tobacco industry increasingly targeting young females in their advertisements. ${ }^{1}$ These developments increase the international importance of our research.

The investigated intervention is easy to implement and can be added to existing school-based programmes. We provide the original posters in high resolution for offset print on our website educationtobacco. org/smokerface-randomized-trial.

\section{CONCLUSION}

Our research has the potential to pave the way for a new form of low-cost and broadly available school-based tobacco prevention in the form of poster campaigns promoting a free app. This protocol has been broadly discussed with experts in the field and novel methods have been developed in order to evaluate this new form of app-based tobacco prevention. Our baseline analysis shows good comparability between the groups at baseline after randomisation and provides new insights into the prevalence of smoking and the use of e-cigarettes among pupils in the 6th and 7th grades in Germany.

\section{Dissemination}

Written informed consent was obtained by the data collectors from both the participants themselves and their parents. All participant information will be stored in locked file cabinets in areas with limited access. Participants' study information will not be released outside of the study without the written permission of the participant. Results will be disseminated at national and international conferences, in peer-reviewed journals, on our websites and throughout the multinational Education Against Tobacco network.

\section{Author affiliations}

${ }^{1}$ Universities of Gießen and Marburg Lung Center (UGMLC); Member of the German Center for Lung Research (DZL), Justus-Liebig-University of Gießen, Gießen, Germany

${ }^{2}$ University of Duisburg-Essen, Essen, Germany

${ }^{3}$ University of Freiburg, Freiburg im Breisgau, Germany

${ }^{4}$ Technical University of Munich, Munich, Germany

${ }^{5}$ University of Bonn, Bonn, Germany

${ }^{6}$ Saarland University Medical Center and Saarland University Faculty of Medicine, Homburg, Germany

${ }^{7}$ Massachusetts General Hospital, Boston, Massachusetts, USA

${ }^{8}$ Pulmonary and Respiratory Critical Care Medicine, Thoraxklinik and Translational Lung Research Center Heidelberg (TLRCH), Member of the German Center for Lung Research (DZL), University of Heidelberg, Heidelberg, Germany

${ }^{9}$ Institute of Occupational Medicine, Social Medicine and Environmental Medicine, Goethe-University, Frankfurt, Germany

${ }^{10}$ Department of Cardiology and Pneumology, University Medical Centre, Göttingen, Germany

${ }^{11}$ Division of Public Health Sciences, Cancer Prevention Research Program, Fred Hutchinson Cancer Research Center, Seattle, Washington, USA

${ }^{12}$ Department of Psychiatry and Psychotherapy, University Hospital of Tübingen, Tübingen, Germany

${ }^{13}$ Department of Government, Faculty of Arts and Sciences, Harvard University, Cambridge, Massachusetts, USA

${ }^{14}$ University of Erlangen-Nuremberg, Erlangen, Germany

${ }^{15}$ University of Calgary, Health Sciences Centre, Calgary, Alberta, Canada

${ }^{16}$ Division of Clinical Epidemiology and Aging Research, German Cancer Research Center (DKFZ), Heidelberg, Germany

Contributors TJB wrote the manuscript, designed all figures, contributed substantially to the development of the questionnaire and supported statistical analysis for the item selection, coordinated and conducted parts of the pilot study, initiated and designed the study, raised the funds, invented, conceived and pretested the posters; invented, conceived and optimised the Smokerface and the Smokerstop app, coordinated the conduct of the study and conducted the baseline analysis and will support outcome analysis. JH organised, coordinated and conducted data collection of 58 schools in the region of Thüringen, implemented the intervention in this region, pretested the questionnaire, conducted the pilot study, contributed to baseline analysis, supported the design of the posters and will conduct statistical analysis. NR and UM proofread the manuscript and made substantial contributions to the design of the study. MK, CB and FH coordinated the data collection of 32 schools in the Rhine-Neckar region of Germany, contributed to the study design and proofread the manuscript. TGB contributed substantially to the development of the questionnaire, conducted the statistical analysis for the item selection, wrote most of the section describing the questionnaire as well as most of the section on the Theory of Planned Behavior (expanded on in the long version of the protocol) and proofread the manuscript. WS and $H G$ support the coordination of the study and proofread the manuscript. TR, RT and DAG contributed to the design of the questionnaire and proofread the manuscript. KS, LJ and HMB coordinated the data collection in 12 schools in Freiburg, implemented the intervention in this region and proofread the manuscript. FNF and HS coordinated the data collection in seven schools in Rhineland-Palatinate, implemented the intervention in this region and proofread the manuscript. MH and AC coordinated the data collection in eight schools in Munich, implemented the intervention in this region and proofread the manuscript. JLS coordinated the data collection in three schools in Bonn, implemented the intervention in this region and proofread the manuscript. VM coordinated the data collection in five schools in Fulda, implemented the intervention in this region and proofread the manuscript. PM contributed to the study design and proofread the manuscript. $A B$ contributed to the design of the study and proofread the manuscript. $\mathrm{CHH}$ contributed to the design of 
the questionnaire and proofread the manuscript. LT coordinated the data collection in two schools in Erlangen, implemented the intervention in this region and proofread the manuscript. FJH substantially contributed to the concept of data entry and proofread the manuscript. AO substantially contributed to the protocol for data collection, critically reviewed the design of the study and proofread the final manuscript.

Funding The Smokerface Randomized Trial was funded by the German Heart Foundation (Bockenheimer Landstraße 94-96, 60323 Frankfurt am Main, Germany). The German Heart Foundation had no role in the design and conduct of the study; collection, management, analysis and interpretation of the data; and preparation, review, or approval of the manuscript

Competing interests There are no financial competing interests with regards to the Smokerface App. The Smokerface App is free and contains no advertising. It was designed for charitable causes.

Ethics approval Ethics committee of the University of Giessen and the ministries of cultural affairs of the six federal states participating in Germany including its amendment to collect cotinine saliva samples.

Provenance and peer review Not commissioned; peer reviewed for ethical and funding approval prior to submission.

Data sharing statement The translated posters for print and details on the development of the questionnaire can be freely accessed (after this manuscript is published) on our website educationtobacco.org/smokerfacerandomized-trial.

Open Access This is an Open Access article distributed in accordance with the Creative Commons Attribution Non Commercial (CC BY-NC 4.0) license, which permits others to distribute, remix, adapt, build upon this work noncommercially, and license their derivative works on different terms, provided the original work is properly cited and the use is non-commercial. See: http:// creativecommons.org/licenses/by-nc/4.0/

\section{REFERENCES}

1. World Health Organization WHO. WHO report on the global tobacco epidemic, 2015. Raising taxes on tobacco, 2015.

2. Haustein KO, Groneberg DA. Chapter 12. In: Tobacco or health?: physiological and social damages caused by tobacco smoking. Springer Science \& Business Media, 2009:360

3. Inchley J, Currie D. Growing up unequal: gender and socioeconomic differences in young people's health and well-being. Health Behaviour in School-aged Children (HBSC) study: international report from the 2013/2014 survey. WHO, 2016. Health policy for children and adolescents 2016(7).

4. Kuntz B, Lampert T. Smoking and passive smoke exposure among adolescents in Germany. Dtsch Arztebl Int 2016;113:23-30

5. Kuntz B, Lampert T. Educational differences in smoking among adolescents in Germany: what is the role of parental and adolescent education levels and intergenerational educational mobility? Int J Environ Res Public Health 2013;10:3015-32.

6. Thomas RE, McLellan J, Perera R. School-based programmes for preventing smoking. Cochrane Database Syst Rev 2013;(4):CD001293.

7. Thomas RE, McLellan J, Perera R. School-based programmes for preventing smoking. Evidence-Based Child Health: A Cochrane Review Journal 2013;8:1616-2040.

8. Burford O, Jiwa M, Carter O, et al. Internet-based Photoaging within Australian pharmacies to promote smoking cessation: randomized controlled trial. J Med Internet Res 2013;15:e64.

9. Weiss C, Hanebuth D, Coda P, et al. Ageing images as a motivational trigger for smoking cessation in young women. Int $J$ Environ Res Public Health 2010;7:3499-512.

10. Brinker TJ, Seeger W, Buslaff F. Photoaging mobile apps in school-based tobacco prevention: the Mirroring Approach. J Med Internet Res 2016;18:e183.

11. McEachan RRC, Conner M, et al. Prospective prediction of health-related behaviours with the Theory of Planned
Behaviour:

a meta-analysis. Health Psychology Review 2011;5:97-144.

12. Mosley JG, Gibbs AC. Premature grey hair and hair loss among smokers: a new opportunity for health education? BMJ 1996;313:1616.

13. Trüeb RM. Association between smoking and hair loss: another opportunity for health education against smoking? Dermatology 2003;206:189-91.

14. Schäfer T, Nienhaus A, Vieluf D, et al. Epidemiology of acne in the general population: the risk of smoking. $\mathrm{Br} J$ Dermatol 2001;145:100-4.

15. Shen Y, Wang T, Zhou C, et al. Prevalence of acne vulgaris in Chinese adolescents and adults: a community-based study of 17,345 subjects in six cities. Acta Derm Venereol 2012; 92:40-4.

16. Okada HC, Alleyne B, Varghai K, et al. Facial changes caused by smoking: a comparison between smoking and nonsmoking identical twins. Plast Reconstr Surg 2013;132:1085-92

17. White V, Bariola E, Faulkner A, et al. Graphic health warnings on cigarette packs: how long before the effects on adolescents wear out? Nicotine Tob Res 2015;17:776-83.

18. Brinker TJ, Seeger W. Photoaging mobile apps: a novel opportunity for smoking cessation? J Med Internet Res 2015;17:e186.

19. Peterson AV Jr, Kealey KA, Mann SL, et al. Hutchinson Smoking Prevention Project: long-term randomized trial in school-based tobacco use prevention-results on smoking. J Natl Cancer Inst 2000;92:1979-91.

20. Van De Ven MO, Engels RC, Otten R, et al. A longitudinal test of the theory of planned behavior predicting smoking onset among asthmatic and non-asthmatic adolescents. J Behav Med 2007;30:435-45.

21. Arrazola RA, Singh T, Corey GC, et al. Tobacco use among middle and high school students-United States, 2011-2014. MMWR 2015;64:381-5.

22. Robine J, Jagger C. Translation \& linguistic evaluation protocol \& supporting material. WHO/UNESCAP Project on Health and Disability Statistics, 2003.

23. Kreuter M, Bauer CM, Ehmann M, et al. [Efficacy and sustainability of a smoking prevention program for pupils-"ohnekippe"]. Dtsch Med Wochenschr 2014;139:1403-8.

24. Brinker TJ, Stamm-Balderjahn S, Seeger W, et al. Education Against Tobacco (EAT): a quasi-experimental prospective evaluation of a programme for preventing smoking in secondary schools delivered by medical students: a study protocol. BMJ Open 2014;4: e004909.

25. Zou G, Donner A. Confidence interval estimation of the intraclass correlation coefficient for binary outcome data. Biometrics 2004;60:807-11.

26. Brinker TJ, Stamm-Balderjahn S, Seeger W, et al. Education Agains Tobacco (EAT): a quasi-experimental prospective evaluation of a multinational medical-student-delivered smoking prevention programme for secondary schools in Germany. BMJ Open 2015;5: e008093.

27. Donald A, Donner A. Adjustments to the Mantel-Haenszel chi-square statistic and odds ratio variance estimator when the data are clustered. Stat Med 1987;6:491-9.

28. Sterne JA, White IR, Carlin JB, et al. Multiple imputation for missing data in epidemiological and clinical research: potential and pitfalls. BMJ 2009;338:b2393.

29. Ybarra ML, Jiang Y, Free C, et al. Participant-level meta-analysis of mobile phone-based interventions for smoking cessation across different countries. Prev Med 2016;89:90-7.

30. Harter S. Causes and consequences of low self-esteem in children and adolescents. In: Self-esteem: the puzzle of low self-regard. edn. Baumeister RF. ed. Boston, MA: Springer US, 1993:87-116.

31. Van De Ven MO, Engels RC, Otten R, et al. A longitudinal test of the Theory of Planned Behavior predicting smoking onset among asthmatic and non-asthmatic adolescents. J Behav Med 2007;30:435-45.

32. Bundeszentrale für gesundheitliche Aufklärung B. Rauchen bei Jugendlichen und Erwachsenen in Deutschland 2014. In: Ergebnisse einer aktuellen Repräsentativbefragung und Trends. Orth B, Töppich J ed. Köln: BZgA, 2015. 